\title{
Lactobacillus brevis CD2 for Prevention of Oral Mucositis in Patients With Head and Neck Tumors: A Multicentric Randomized Study
}

\author{
VITALIANA DE SANCTIS ${ }^{1}$, LILIANA BELGIOIA ${ }^{2}$, DOMENICO CANTE ${ }^{3}$, MARIA R. LA PORTA ${ }^{3}$, \\ ORIETTA CASPIANI ${ }^{4}$, ROBERTA GUARNACCIA ${ }^{4}$, ANGELA ARGENONE ${ }^{5}$, PAOLO MUTO ${ }^{5}$, \\ DANIELA MUSIO ${ }^{6}$, FRANCESCA DE FELICE ${ }^{6}$, FRANCESCA MAURIZI ${ }^{7}$, FEISAL BUNKHELIA $^{7}$, \\ MARIA G. RUO REDDA ${ }^{8}$, ALESSIA REALI ${ }^{8}$, MAURIZIO VALERIANI ${ }^{1}$, MATTIA F. OSTI ${ }^{1}$, \\ DANIELA ALTERIO ${ }^{9}$, ALMALINA BACIGALUPO $^{10}$ and ELVIO G. RUSSI ${ }^{11}$ \\ ${ }^{I}$ Department of Medicine and Surgery and Translational Medicine, Radiation Oncology, \\ S. Andrea Hospital, Sapienza University of Rome, Rome, Italy; \\ ${ }^{2}$ Radiation Oncology, Policlinico San Martino and University, Genoa, Italy; \\ ${ }^{3}$ Department of Radiation Oncology, ASL Turin 4, Ivrea Community Hospital, Ivrea, Italy; \\ ${ }^{4}$ Radiation Oncology, Fatebenefratelli Hospital, Rome, Italy; \\ ${ }^{5}$ Radiation Oncology, Istituto Nazionale Tumori-IRCCS-G. Pascale Foundation, Naples, Italy; \\ ${ }^{6}$ Department of Radiological, Oncological and Anatomo-pathological Sciences, \\ Radiation Oncology, Policlinico Umberto I, Sapienza University of Rome, Rome, Italy; \\ ${ }^{7}$ Radiation Oncology, A.O. Ospedali Riuniti Marche Nord, Pesaro, Italy; \\ ${ }^{8}$ Department of Oncology, Radiation Oncology, University of Turin, S. Luigi Gonzaga Hospital, Orbassano, Italy; \\ ${ }^{9}$ Radiation Oncology, European Institute of Oncology, Milan, Italy; \\ ${ }^{10}$ Radiation Oncology, Policlinico San Martino, Genoa, Italy; \\ ${ }^{11}$ Radiation Oncology, A.S.O. S. Croce e Carle, Cuneo, Italy
}

\begin{abstract}
Background: Oropharyngeal mucositis occurs in virtually all patients with head and neck cancer receiving radiochemotherapy. The manipulation of the oral cavity microbiota represents an intriguing and challenging target. Patients and Methods: A total of 75 patients were enrolled to receive Lactobacillus brevis CD2 lozenges or oral care regimen with sodium bicarbonate mouthwashes. The primary endpoint was the incidence of grade 3 or 4 oropharyngeal mucositis during radiotherapy treatment. Results: There was no statistical difference in the incidence of grade 3-4 oropharyngeal mucositis between the intervention and control groups $(40.6 \%$ vs. $41.6 \%$ respectively, $p=0.974)$. The incidence of pain, dysphagia, body weight loss and quality of life were
\end{abstract}

This article is freely accessible online.

Correspondence to: Vitaliana De Sanctis, Department of Medicine and Surgery and Translational Medicine, Radiation Oncology, S. Andrea Hospital, Sapienza University of Rome, via di Grottarossa 1035, Rome, Italy. Tel: +390633776160, e-mail: vitaliana. desanctis@uniroma1.it

Key Words: Lactobacillus brevis CD2, mucositis, head neck cancer, IMRT, radiochemotherapy, radiobiotherapy. not different between the experimental and standard arm. Conclusion: Our study was not able to demonstrate the efficacy of L. brevis CD2 lozenges in preventing radiation-induced mucositis in patients with head and neck cancer. Although modulating homeostasis of the salivary microbiota in the oral cavity seems attractive, it clearly needs further study.

Acute radiation-related toxicities represent a clinicallyrelevant problem during curative radiotherapy (RT) for patients with head and neck cancer (HNC). Among different side-effects, oropharyngeal mucositis (OM) remains one of the most important issues for patients with HNC, with a negative impact on their quality of life (QoL), and also on locoregional control due to the need for treatment breaks that extend the planned treatment time (1-6).

The development of OM is complex and begins from clonogenic death of basal stem cells due to DNA strand breaks caused by reactive oxygen species (7). Through the complex activation of several transcription factors, it seems to end with the production of pro-inflammatory cytokines that promote and amplify cellular damage to the oral mucosa (8). Despite recent improvements in our understanding of these processes, preventive and therapeutic management of $\mathrm{OM}$ is still a debated and open question. A large number of 
agents, both natural and pharmacological, have been tested with inconsistent results. To date, only oral care protocols were recommended by the Mucositis Study Group of the Multinational Association of Supportive Care in Cancer and International Society of Oral Oncology and others as a validated approach in the prevention of OM in patients with HNC treated with curative RT (9-11).

The oral cavity is one of the most biologically complex environments of the human body and the role of the resident microflora in OM development during RT is still debated. To date, interventional strategies for mucositis that focused on manipulation of the oral cavity microbiota have been substantially unsuccessful (12-16). Many bacteria are implicated in the pathogenesis and maintenance of inflammatory diseases of the oral cavity (periodontal disease, Behcet's disease, recurrent aphthous stomatitis) and most of them require arginine for their survival and their potential pathogenicity (17-19).

Recently, Sharma et al. reported a statistically significant reduction of grade 3-4 OM related to the use of Lactobacillus brevis CD2 lozenges (LB CD2) during radiochemotherapy (RCHT) in patients with HNC (20). L. brevis $\mathrm{CD} 2$, which produces high levels of arginine deaminase, is able to reduce arginine availability in the oral cavity and, therefore, reduces arginine-dependent growth of microorganisms implicated in inflammatory processes there. Moreover, LB CD2 reduces arginine availability for arginases, resulting in a reduction of nitrous oxide production and a subsequent attenuation of inflammatory processes.

Here we present the results of an Italian multi-centric, prospective randomized trial designed to compare LB CD2 versus sodium bicarbonate mouthwash in patients with $\mathrm{HNC}$ treated with RCHT or biological agent-radiotherapy (bioRT). The aim of the study was to verify the beneficial effects of LB CD2 in the prevention of OM onset during the RT course.

\section{Patients and Methods}

This was a multicentric, phase III, open-label, randomized controlled trial, conducted by eight Italian Oncological Radiotherapy Departments between April 2012 and July 2015. This study received approvals from the local clinical research Ethics Committee and was conducted in full accordance with Helsinki declaration (Clinical Trials number NCT01707641). All the patients gave their informed consent.

Inclusion criteria were: Histological diagnosis of head and neck carcinoma (except larynx, parotid and other salivary glands tumors); age of 18 years or above; concomitant treatment with RCHT or bio$\mathrm{RT}$ in a radical setting; baseline Karnofsky performance status $>70$; normal bone marrow, renal, and liver functions. Exclusion criteria were: Inability to use mouthwash; any previous RT to the head and neck region; major surgery approach exceeding biopsy within 4 weeks prior to the enrollment; contrast enhancement computed tomography or positron-emission tomography for distant metastasis detection; mucosal ulceration at baseline due to viral or mycotic chronic disease; anti-mycotic/antiviral drugs within 1 week of treatment start; comorbidities (insulin-dependent diabetes mellitus; hepatitis B or C); signs and symptoms of active systemic or dental infection.

All patients were treated with intensity-modulated radiation therapy (IMRT). Dose fractionation was delivered with a prescribed total dose of 68-70 Gy and 50-54 Gy to the macroscopic disease and low-risk regions, respectively. Concomitant cisplatinum-based chemotherapy was administered using a weekly $\left(40 \mathrm{mg} / \mathrm{m}^{2}\right)$ or a 3 weekly $\left(100 \mathrm{mg} / \mathrm{m}^{2}\right)$ schedule. Cetuximab was administered concurrently with RT according to Bonner's schedule (21). The choice of systemic concomitant therapy was at discretion of the attending physicians. Neo-adjuvant chemotherapy (docetaxel, cisplatin and 5-fluorouracil every 21 days for three cycles) was allowed for patients with nasopharyngeal cancer.

Prior to starting RCHT or bioRT, enrolled patients were randomised (1:1) to standard oral care regimen with sodium bicarbonate mouthwash (control arm) or LB CD2 lozenges (intervention arm) through a computer-generated randomization list consisting of randomly permuted blocks of four patient numbers.

The LB CD2 lozenges contained not less than $2 \times 10^{9}$ viable cells of L. brevis $\mathrm{CD} 2$ as the active ingredient. The LB CD2 lozenges were supplied by CD Investments Ltd, Rome, Italy. The daily dose was six lozenges per day, one every $2-3 \mathrm{~h}$ to be dissolved in the mouth and then swallowed. Hot beverages (e.g. tea, coffee, or milk) were avoided for at least half an hour before and after administration, as high temperature reduces the efficacy of LB CD2. The patients in the intervention arm were to receive LB CD2 lozenges from the first day of RT up to 1 week after the end of treatment. Patients in the control arm received sodium bicarbonate mouthwash at least three times a day from the first day of RT until the end of treatment.

If patients reported grade 3-4 OM, LB CD2 tablets or standard prophylaxis was stopped and patients started symptomatic local with or without systemic therapies at the discretion of the single center.

OM grade was registered weekly during the RT course by trained physicians according to the NCI Common Toxicity Criteria scoring system (CTCAE) version 4.0 (22).

QoL was assessed using the FACT H\&N (version 4.0) quality of life questionnaire, at the following time points: Pre-RT, at the end of RT, and at 1, 3, 6, 9 and 12 months from the end of RCHT or bioRT (23). Pain and dysphagia were weekly recorded during radiotherapy by Numeric Rating Scale and according to Radiation Therapy Oncology Group Common Toxicity Criteria, and at 1, 3, 6, 9 and 12 months from the end of RCHT or bioRT, respectively (24).

All clinical data were collected prospectively with weekly clinic visits during RT and at 1, 3, 6, 9 and 12 months after the end of treatment.

Statistical analysis. The primary endpoint of our study was to compare the incidence of severe OM between the study group (LB $\mathrm{CD} 2 \mathrm{arm}$ ) and the control group (sodium bicarbonate mouthwash arm) of patients with HNC treated with curative intent.

Secondary endpoints included: a) Number of patients who were able to complete the planned treatment; b) requirement for enteral nutrition; c) incidence and severity of treatment-related dysphagia; d) patient quality of life; e) body weight loss during the treatment course; f) the incidence and time-course of treatment-related pain. The statistical analysis for the primary and secondary endpoints was based on the intention-to-treat population. 
We assumed observation of severe (grade 3 and 4) OM incidence in $75 \%$ of patients treated with sodium bicarbonate. In the group of patients treated with LB CD2, we expected a $50 \%$ incidence of mucositis, which was lower by $25 \%$ compared to that of the reference group, with an odds ratio $(\mathrm{OR})$ of 0.333 . In order to detect a statistically significant effect with an OR of 0.333 with $\alpha=0.05$ (one-tailed) and $\beta=0.20$ (power equal to $80 \%$ ), it was necessary to enroll a total of 92 patients. Considering a patient drop out rate of $10-15 \%$, we were required to enroll at least 106 patients (53 patients randomized to each of the two treatment groups).

Tumor stage and types of concomitant treatment (cisplatin, cetuximab or neoadjuvant chemotherapy) were analyzed according to a stepwise logistic regression analysis.

Parametric variables were analyzed using Student's $t$-test for independent data, while the non-parametric or non-normally distributed variables were analyzed by Mann-Whitney $U$-test.

Discrete variables, nominal or categoricaI were evaluated by the chi-square test.

The data were analyzed by using the SAS statistical software version 9.4 (Cary, NC, USA). $p$-Values of 0.05 or less were considered statistically significant.

\section{Results}

From April 2012 to July 2015, a total of 75 patients were enrolled. The enrollment was prematurely stopped without achieving the planned accrual of 106 patients because the probiotic lozenges were no longer available for the study due to technical and administrative problems in product supply by the company. Seven out of 75 patients were excluded from the statistical analysis (four were considered ineligible and were not randomized; three randomized patients presented too high a number of missing data for all the records available) and consequently the analysis was performed on a sample size of 68 patients (32 in the intervention arm and 36 in the control arm).

Patients characteristics are shown in Table I. Baseline patient characteristics were similar for the two population arms for age, gender, body mass index and clinical tumor stage. On the contrary, we recorded more cases of nasopharyngeal and hypopharyngealgeal cancer in the intervention group and more oropharyngeal cancer in the control group.

Nearly $80 \%$ of patients underwent cisplatinum-based RCHT, most of them with $100 \mathrm{mg} /$ cisplatin every 3 weeks: the weekly schedule (40 mg/m $\mathrm{m}^{2}$ cisplatin) was used in $6 / 27$ $(22 \%)$ patients in the intervention group and $3 / 23(13 \%)$ patients in the control group.

All patients took the LB CD2 lozenges or sodium bicarbonate mouthwash at the planned dose, until the occurrence of grade 3-4 OM or until the occurrence of nausea or major clinical complications that needed systemic supportive care. Fifteen patients discontinued the lozenges during treatment: 11 due to lozenge-induced nausea within 2 weeks of starting treatment and four due to the occurrence of complications that required systemic antibiotic therapy (bleeding, pneumonia, sepsis).
There was no serious adverse event related to LB CD2 lozenge administration.

Two early treatment-related deaths were recorded: one due to acute renal failure (experimental arm) and one due to acute respiratory distress syndrome (control arm).

Results showed no difference in the incidence of severe OM between the intervention and control groups $(40.6 \%$ versus $41.6 \%$ respectively, $p=0.974$ ) during the RT course (Table II).

From the analysis of the clinical and tumor-related variables, a significant association was recorded only for $\mathrm{T}$ stage, regardless of the randomization arm. Patients with stage T1-T3 had a statistically significant reduction of risk of developing OM than patients with $\mathrm{T} 4$ stage $(\mathrm{OR}=0.164$, $95 \%$ confidence interval $=0.040-0.673 ; p<0.05)$.

The systemic treatments categorized as neoadjuvant chemotherapy or concurrent chemotherapy or concurrent biotherapy did not have an impact on the development of severe OM with respect to the randomization arm.

The QoL score was studied considering the feedback collected from self-reported survey at baseline, at the end of treatment and during follow-up. All but six patients completed the QoL questionnaires during treatment, 62 patients at 1 month, 53 at 3 months, 52 at 6 months, 50 at 9 months and 42 at 12 months. These data were analyzed using variance analysis for repeated measures. The time trend of quality of life score was statistically different $(p<0.05)$ among the study phases (baseline, treatment course, follow-up). Indeed, the QoL score tended to worsen during the treatment course compared to the baseline condition, while during the follow-up period, the QoL tended to recover to the baseline level (Figure 1). No effect due to the experimental treatment or concurrent chemotherapy was observed. Since the number of patients who received concurrent cetuximab was very limited, it is not possible to draw any conclusions on the different impact on OM occurrence of these two different concomitant systemic approaches

A statistically significant $(p<0.01)$ tendency for weight loss during concurrent therapy compared to the baseline was recorded, independently of the experimental or standard arm.

The time course of pain was statistically significant $(p<0.01)$. Results showed that pain increased during the treatment phase, while in the follow-up phase it tended to recover to baseline values, with no difference in the experimental treatment or standard arms (Figure 2).

Regarding dysphagia, we did not observe statistically significant differences between the two arms. On the contrary, we recorded a statistically significant risk reduction for enteral nutrition needs for the standard arm compared to the experimental arm $(\mathrm{OR}=0.341,95 \%$ confidence intervaI $=0.127$ $0.917 ; p<0.05$ ) (Table III). Finally, the percentage of patients who completed concomitant treatment did not differ significantly between the two groups of patients. 
Table I. Characteristics of patients at baseline.

\begin{tabular}{|c|c|c|c|}
\hline & LB CD2 (n=32) & Na Bicarbonate $(n=36)$ & All patients $(n=68)$ \\
\hline \multicolumn{4}{|l|}{ Gender, n (\%) } \\
\hline Male & $26(81.2)$ & $27(75)$ & $53(77.9)$ \\
\hline Female & $6(18.8)$ & $9(25)$ & $15(22.1)$ \\
\hline Mean age (range), years & $58.4(34-74)$ & $60(39-77)$ & $60(57.3-62.5)$ \\
\hline Mean BMI (range), $\mathrm{kg} / \mathrm{m}^{2}$ & $25.6(14.8-35.2)$ & $25.1(16.2-40.1)$ & $25.2(14.8-40.1)$ \\
\hline \multicolumn{4}{|l|}{ Primary site, $\mathrm{n}(\%)$} \\
\hline Unknown & $3(9.4)$ & $1(2.8)$ & $4(5.9)$ \\
\hline $\mathrm{T} 1$ & $1(3.1)$ & $2(5.6)$ & $3(4.4)$ \\
\hline $\mathrm{T} 2$ & $13(40.6)$ & $8(22)$ & $21(30.9)$ \\
\hline $\mathrm{T} 3$ & $7(21.9)$ & $12(33.3)$ & $19(27.9)$ \\
\hline T4 & $8(25)$ & $13(36.1)$ & $21(30.9)$ \\
\hline \multicolumn{4}{|l|}{ Nodal involvement, n (\%) } \\
\hline No & $3(9.4)$ & $2(5.6)$ & $5(7.4)$ \\
\hline $\mathrm{N} 1$ & $3(9.4)$ & $8(22.2)$ & $11(16.2)$ \\
\hline $\mathrm{N} 2$ & $25(78.1)$ & $25(69.4)$ & $50(73.5)$ \\
\hline N3 & $1(3.1)$ & $1(2.8)$ & $2(2.9)$ \\
\hline \multicolumn{4}{|l|}{ TNM stage, n (\%) } \\
\hline IIA & $2(6.3)$ & $0(0)$ & $2(2.9)$ \\
\hline III & $5(15.6)$ & $5(13.9)$ & $10(14.7)$ \\
\hline IVA & $21(65.6)$ & $24(66.7)$ & $45(66.2)$ \\
\hline IVB & $1(3.1)$ & $4(11.1)$ & $5(7.4)$ \\
\hline Not evaluable & $3(9.4)$ & $3(8.3)$ & $6(8.8)$ \\
\hline Cisplatin, $\mathrm{n}(\%)$ & $27(84.4)$ & $26(72.2)$ & $53(77.9)$ \\
\hline Cetuximab, n (\%) & $3(9.4)$ & $4(11.1)$ & $7(10.3)$ \\
\hline Neoadjuvant CHT, n (\%) & $2(6.2)$ & $6(16.7)$ & $8(11.8)$ \\
\hline
\end{tabular}

CHT: Chemotherapy; BMI: body mass index; LB CD2: Lactobacillus brevis CD2 lozenges.

\section{Discussion}

We conducted a prospective randomized study to compare the incidence of OM between patients treated with LB CD2 lozenges and patients treated with sodium bicarbonate mouthwash during radical concurrent RCHT or bio-RT for HNC. Bearing in mind the statistical limitation of the present study (the planned accrual was not reached due to the lack of LB CD2 supply), our results did not demonstrate any benefit of $\mathrm{LB} \mathrm{CD} 2$ in reducing the incidence of severe $\mathrm{OM}$ (intervention versus control arm: $40.6 \%$ versus $41.6 \%$, respectively). Moreover, patients enrolled in the experimental arm did not have any significant improvement of QoL or acute toxicities such as weight loss, pain and dysphagia. Our study has not confirmed the promising results obtained by Sharma et al. (20). Sharma et al. showed that patients in the LB CD2 arm developed a significant lower rate of grade 3 and 4 OM than those in the placebo arm $(52 \%$ versus $77 \%, p<0.001)$.

Obviously, the main bias of our study is represented by a sample size lower than that planned. On the other hand, there are several differences between the present study and that of Sharma et al. which could partly explain our different results. Firstly, IMRT was used for all our patients, while a 2D technique was employed in Sharma et al.'s study.
Table II. Incidence of severe oral mucositis (OM) between the intervention and control groups.

\begin{tabular}{lccc}
\hline & \multicolumn{2}{c}{ Grate 3-4 OM, n (\%) } & \\
\cline { 2 - 3 } Group & Yes & No & Total, n (\%) \\
\hline LB CD2 & $13(40.6 \%)$ & $19(59.3 \%)$ & $32(47.1 \%)$ \\
Control & $15(41.6 \%)$ & $21(58.3 \%)$ & $36(52.9 \%)$ \\
Total & $28(41.2 \%)$ & $40(58.8 \%)$ & $68(100 \%)$ \\
\hline
\end{tabular}

LB CD2: Lactobacillus brevis CD2 lozenges.

Table III. Incidence of requirement for enteral nutrition between the intervention and control groups.

\begin{tabular}{lrrl}
\hline & \multicolumn{2}{c}{ Enteral nutrition, n (\%) } & \\
\cline { 2 - 3 } Group & \multicolumn{1}{c}{ Yes } & No & Total, n (\%) \\
\hline LB CD2 & $12(37,5 \%)$ & $20(62.5 \%)$ & $32(47 \%)$ \\
Control & $6(16.6 \%)$ & $30(83.3 \%)$ & $36(53 \%)$ \\
Total & $18(26.5 \%)$ & $50(73.5 \%)$ & $68(100 \%)$ \\
\hline
\end{tabular}

LB CD2: Lactobacillus brevis CD2 lozenges. 


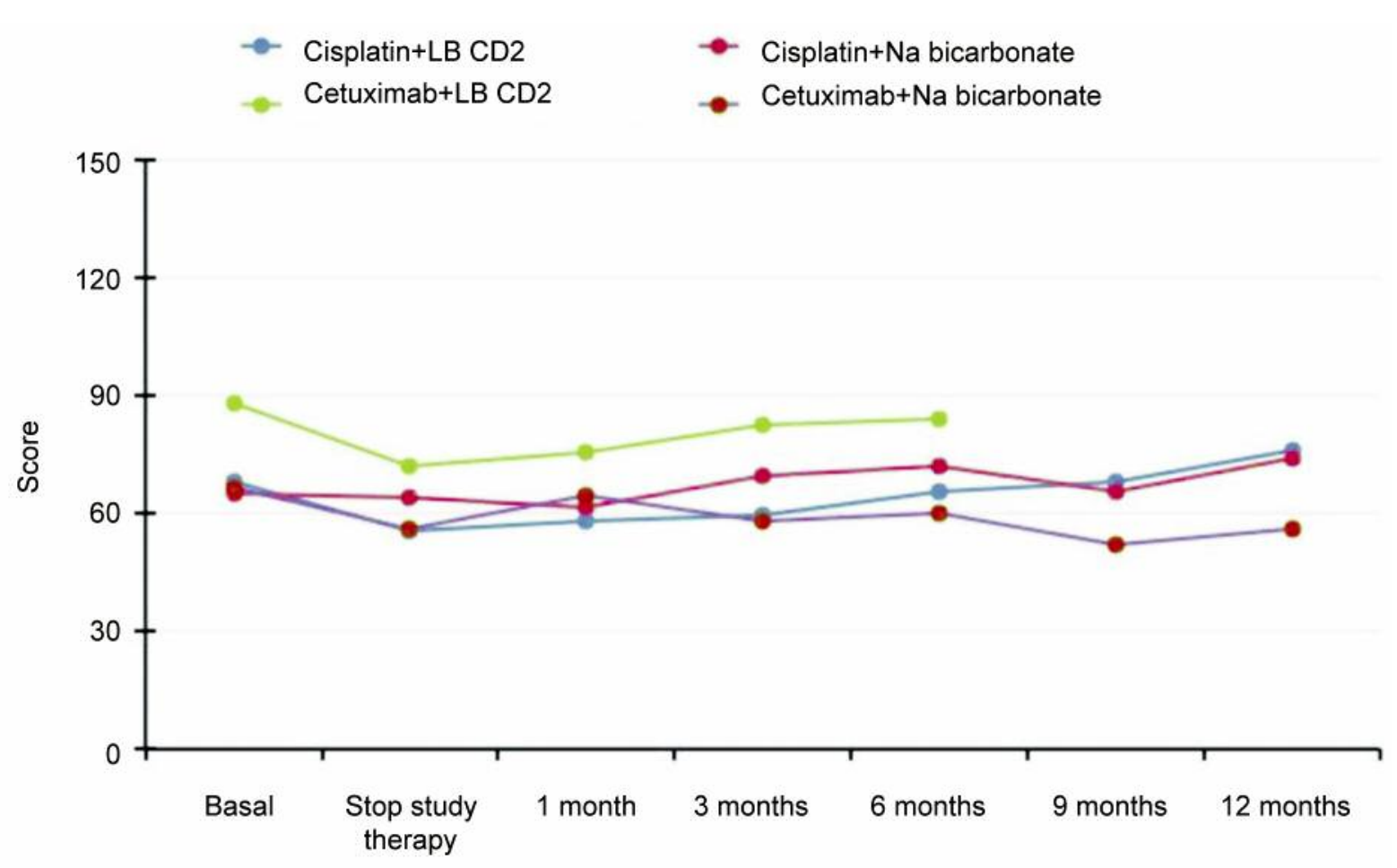

Figure 1. The variation of the quality of life score for each treatment arm and chemotherapy schedule during treatment and follow-up. LB CD2: Lactobacillus brevis CD2 lozenges.

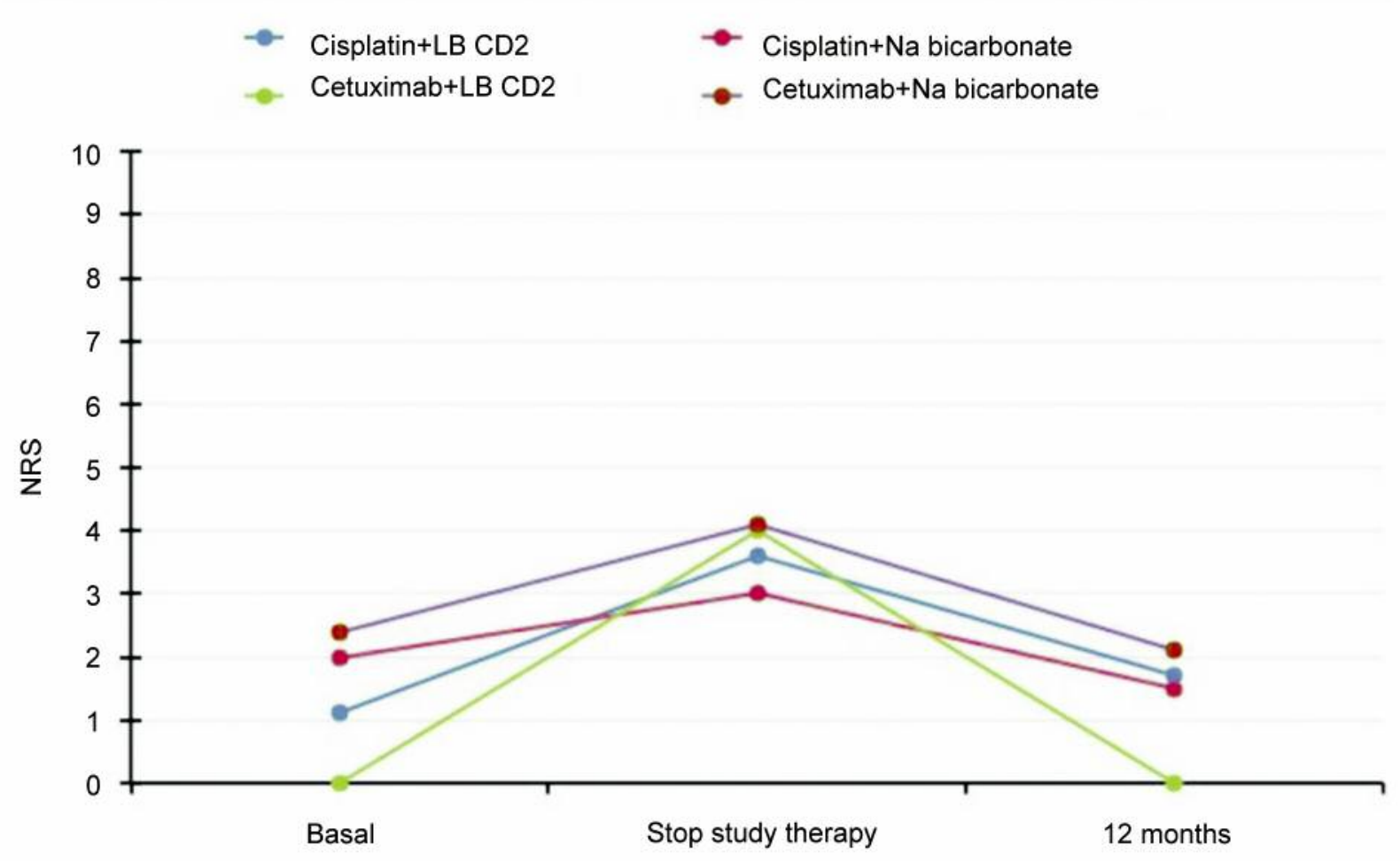

Figure 2. The variation of the NRS assessment for each treatment arm and chemotherapy schedule during treatment and follow-up. LB CD2: Lactobacillus brevis CD2 lozenges; NRS: numeric rating scale (pain). 
Nowadays, IMRT represents the best technique to improve long-term salivary flow rates with a positive impact on QoL $(2,11,25)$. Therefore, IMRT may have improved the oral mucosal tolerance to RCHT or bioRT in our patients, reducing the possible beneficial impact of $\mathrm{LB} C \mathrm{CD} 2$ in our experimental arm. Recently, interesting speculations have been made about the role of IMRT in reducing the rate of grade 3-4 OM, sparing the oral mucosa outside the planned target volume and therefore reducing overall short- and longterm morbidity $(26,27)$. Although the sparing of the oral mucosa with IMRT seems a promising and attractive approach in order to reduce the severity of acute mucositis and improving QoL, the definition and contouring of the volume of the oral mucosa in a reproducible and consistent way still appears problematic (28).

Radiation doses received by the mucosa of the oral cavity and oropharynx were not recorded in our multicentric study, hence this may be another limitation of our results.

The analysis of the clinical and tumor-related variables showed a significant association only for the T-stage, regardless of the randomization arm. In particular, patients with stage $\mathrm{T} 4$ had a statistically significantly greater risk of developing OM than patients with T1-T3 stage. This may be explained by the clinical target/planned target volumes in patients with T4 tumor being supposedly larger than in those with T1-T3, although we had not recorded these data.

Furthermore, our control-arm group received sodium bicarbonate mouthwash as recommended in several guidelines, while the control group of Sharma et al. was enrolled to receive placebo lozenges $(9-11,20)$.

Therefore, different RT techniques and different preventive management may explain the lower rate of severe OM incidence in our control group (41.6\%) compared to Sharma et al.'s study (77\%).

In our experience, LB CD2 was not related to a beneficial effect on body weight, pain and quality of life. In fact, all these endpoints showed similar deterioration in the intervention and control arms during treatment. As expected, all the patients experienced symptom regression during the first year of follow-up, with improvement also of QoL parameters, without significant differences between the two groups. On the contrary, patients enrolled in the standardtreatment arm had a statistically significant lower risk of enteral nutrition $(p<0.05)$ compared to the experimentaltreatment arm. However, this difference should be cautiously considered because it may reflect different enteral nutrition supply approaches and the inhomogeneous assessment strategies adopted by the Italian RT Departments rather than a true statistical difference.

We recorded interruptions of $\mathrm{LB}$ CD2 lozenge intake in $11 / 32$ patients due to unpleasant taste of the lozenges. All our patients underwent concomitant chemotherapy or biotherapy and, therefore, were treated with antiemetic drugs. Although antiemetic therapy was modulated to correct increasing nausea, $34.3 \%$ of patients discontinued taking LB CD2.

Despite our results, the modulation of oral cavity environment remains a promising field because the salivary microbiota plays an important role in homeostasis of the oral cavity (13-14). At present, studies that demonstrated efficacy in modulating the salivary microbiota were mainly focused on non-neoplastic diseases of the oral cavity. In this setting, a favorable microbiota balance and a recovery of a healthy oral environment are more easily achieved, allowing resolution of the disease $(17-19,29)$. On the contrary, controversial results have been shown between the presence/manipulation of specific bacterial species and mucositis occurrence in patients treated with radiotherapy for HNC. Many differences between in bacterial strains studied, study population, collection time, sampling methods, and scoring methods for mucositis make it impossible to draw any conclusions from these studies $(15,16$, 30-32). Nevertheless, the concept that disease conditions such as OM may be associated with a shift in the microbial composition of the biofilm that colonizes the oral cavity continues to attract more and more attention. In fact, the alteration of the salivary bacterial flora due to tumor itself or RCHT may contribute to mucosal damage, modulating the levels of opportunistic bacteria that can turn pathogenic in patients with cancer who develop $\operatorname{OM}(8,14)$. There is also much emphasis on the role of conditions of other oral sites such as supra- and subgingival periodontitis, although few studies have looked into these conditions $(33,34)$.

$\mathrm{OM}$ induced by RT is, therefore, a complex process that must be tackled from various therapeutic perspectives. Salivary microbiota may represent an intriguing therapeutic perspective and be worthy of further research.

In conclusion, while considering that the statistical power of the study suffered due to the premature closure of patient accrual, we were not able to confirm the beneficial effects of L. brevis CD2 in reducing the rate of grade 3-4 RT-induced $\mathrm{OM}$ in patients with HNC.

Further larger prospective studies are required to explore the impact of modulation of the oral cavity environment in reducing the incidence and severity of $\mathrm{OM}$ in this setting of patients.

\section{Conflicts of Interest}

All relationships or interests that might have had direct or potential influence or impart bias on the work were excluded. The Authors deny any kind of conflict of interest in regard to this study.

\section{Authors' Contributions}

The study was conceived by VDS. All Authors participated in the preparation of the manuscript and contributed to initial drafts, edited version, and final version. All the Authors read and approved the final version before submission. 


\section{References}

1 Tribius S and Bergelt C: Intensity-modulated radiotherapy versus conventional and 3D conformal radiotherapy in patients with head and neck cancer: Is there a worthwhile quality of life gain? Cancer Treat Rev 37: 511-519, 2011. PMID: 21324605. DOI: 10.1016/j.ctrv.2011.01.004

2 Scott-Brown M, Miah A, Harrington $\mathrm{K}$ and Nutting C: Evidence-based review: Quality of life following head and neck intensity-modulated radiotherapy. Radiother Oncol 97: 249-257, 2010. PMID: 20817284. DOI: 10.1016/j.radonc.2010.08.004

3 Elting LS, Keefe DM, Sonis ST, Garden AS, Spijkervet FK, Barasch A, Tishler RB, Canty TP, Kudrimoti MK and Vera-Llonch M: Patient-reported measurements of oral mucositis in head and neck cancer patients treated with radiotherapy with or without chemotherapy: demonstration of increased frequency, severity, resistance to palliation, and impact on quality of life. Cancer 113 : 2704-2713, 2008. PMID: 18973181. DOI: 10.1002/ cncr.23898

4 Elting LS, Cooksley CD, Chambers MS and Garden AS: Risk, outcomes and costs of radiation-induced oral mucositis among patients with head-and-neck malignancies. Int J Radiat Oncol Biol Phys 68: 110-1120, 2007. PMID: 17398022. DOI: 10.1016/ j.ijrobp.2007.01.053

5 Kouloulias V, Thalassinou S, Platoni K, Zygogianni A, Kouvaris J, Antypas C, Efstathopoulos E and Nikolaos K: The treatment outcome and radiation-induced toxicity for patients with head and neck carcinoma in the IMRT era: A systematic review with dosimetric and clinical parameters. BioMed Res Int 2013: 401261, 2013. PMID: 24228247. DOI: 10.1155/2013/401261

6 Russo G, Haddad R, Posner M and Machtay M: Radiation treatment breaks and ulcerative mucositis in head and neck cancer. Oncologist 13: 886-898, 2008. PMID: 18701763. DOI: 10.1634/theoncologist.2008-0024

7 Sonis ST: The pathobiology of mucositis. Nat Rev Cancer 4: 277-84, 2004. PMID: 18701763. DOI: $10.1038 / \mathrm{nrc} 1318$

8 Al-Dasooqi N: Emerging evidence on the pathobiology of mucositis. Support Care Cancer 21: 2075-2083, 2013. PMID: 23842598. DOI: $10.1007 / \mathrm{s} 00520-013-1900-\mathrm{x}$

9 McGuire DB, Fulton JS, Park J, Brown CG, Correa MEP, Eilers J, Eilers J, Elad S, Gibson F, Oberle-Edwards LK, Bowen J and Lalla RV: Systematic review of basic oral care for the management of oral mucositis in cancer patients. Support Care Cancer 21: 3165-77, 2013. PMID: 24018908. DOI: 10.1007/ s00520-013-1942-0

10 Lalla RV, Bowen J, Barasch A Elting L, Epstein J, Keefe DM, McGuire DB, Migliorati C, Nicolatou-Galitis O, Peterson DE, Raber-Durlacher JE, Sonis ST and Elad S: MASCC/ISOO clinical practice guidelines for the management of mucositis secondary to cancer therapy. Cancer 120: 1453-1461, 2014. PMID: 24615748. DOI: 10.1002/cncr.28592

11 De Sanctis V, Bossi P, Sanguineti G, Trippa F, Ferrari D, Bacigalupo A, Ripamonti CI, Buglione M, Pergolizzi S, Langendjik JA, Murphy B, Raber-Durlacher J, Russi EG and Lalla RV: Mucositis in head and neck cancer patients treated with radiotherapy and systemic therapies: Literature review and consensus statements. Crit Rev Oncol Hematol 100: 147-166, 2016. PMID: 26947812. DOI: 10.1016/j.critrevonc.2016.01.010

12 Donnelly JP, Bellm LA, Epstein JB, Sonis ST and Symonds RP: Antimicrobial therapy to prevent or treat oral mucositis. Lancet Infect Dis 3: 405-412, 2003. PMID: 12837345.
13 Stringer AM and Logan RM: The role of oral flora in the development of chemotherapy-induced oral mucositis. J Oral Pathol Med 44: 81-87, 2015. PMID: 24494824. DOI: 10.1111/ jop. 12152

14 Laheij AM and de Soet JJ: Can the oral microflora affect oral ulcerative mucositis? Curr Opin Support Palliat Care 8: 180-187, 2014. PMID: 24743299. DOI: 10.1097/SPC.0000000000000053

15 Stokman MA, Spijkervet FK, Burlage FR, Dijkstra PU, Manson WL, de Vries EG, Roodenburg JL: Oral mucositis and selective elimination of oral flora in head and neck cancer patients receiving radiotherapy: A double-blind randomised clinical trial. Br J Cancer 88: 1012-1016, 2003. PMID: 12671696. DOI: 10.1038/sj.bjc.6600824

16 Nagy K, Urban E, Fazekas O, Thurzo L and Nagy E: Controlled study of lactoperoxidase gel on oral flora and saliva in irradiated patients with oral cancer. J Craniofac Surg 18: 1157-64, 2007. PMID: 17912104. DOI: 10.1097/scs.0b013e3180de6311

17 Bank I, Duvdevani M and Livneh A: Expansion of gammadelta T-cells in Behçet's disease: Role of disease activity and microbial flora in oral ulcers. J Lab Clin Med 141: 33-40, 2003. PMID: 12518166. DOI: $10.1067 / \mathrm{mlc} .2003 .1$

18 Janssen KM, Vissink A, de Smit MJ Westra J and Brouwer E: Lessons to be learned from periodontitis. Curr Opin Rheumatol 25: 241-247, 2013. PMID: 23370377. DOI: 10.1097/ BOR.0b013e32835d833d

19 Riccia DN, Bizzini F, Perilli MG, Polimeni A, Trinchieri V, Amicosante G and Cifone MG: Anti-inflammatory effects of Lactobacillus brevis (CD2) on periodontal disease. Oral Dis 13: 376-385, 2007. PMID: 17577323. DOI: 10.1111/j.16010825.2006.01291.x

20 Sharma A, Rath G.K., Chaudhary S.P, Thakar A, Mohanti BK, Bahadur S: Lactobacillus brevis $\mathrm{CD} 2$ lozenges reduce radiation and chemotherapy-induced mucositis in patients with head and neck cancer: A randomized double-blind placebo-controlled study. Eur J Cancer 48: 875-881, 2012. PMID: 21741230. DOI: 10.1016/j.ejca.2011.06.010

21 Bonner JA, Harari PM, Giralt J, Azarnia N, Shin DM, Cohen RB, Jones CU, Sur R, Raben D, Jassem J, Ove R, Kies MS, Baselga J, Youssoufian H, Amellal N, Rowinsky EK and Ang KK: Radiotherapy plus cetuximab for squamous-cell carcinoma of the head and neck. N Engl J Med 6: 567-578, 2006. PMID: 16467544. DOI: 10.1056/NEJMoa053422

22 CTCAE Panel: Common Terminology Criteria for Adverse Events v4.0 (CTCAE). US Department Health Hum Serv Natl Inst Health, Bethesda National Cancer Institute 2015.

23 List MA, D'Antonio LL, Cella DF, Siston A, Mumby P, Haraf D and Vokes E: The performance status scale for head and neck cancer patients and the functional assessment of cancer therapyhead and neck scale. A study of utility and validity. Cancer 77(11): 2294-2301, 1996. PMID: 8635098. DOI: 10.1002/(SICI) 1097-0142(19960601)77:11<2294::AID-CNCR17>3.0.CO;2-S

24 Cox JD, Stetz J and Pajak TF: Toxicity criteria of the Radiation Therapy Oncology Group (RTOG) and the European Organization for Research and Treatment of Cancer (EORTC). Int J Radiat Oncol Biol Phys 31(5): 1341-1346, 1995. PMID: 7713792. DOI: 10.1016/0360-3016(95)00060-C

25 Nutting CM, Morden JP, Harrington KJ, Urbano TG, Bhide SA, Clark C, Miles EA, Miah AB, Newbold K, Tanay M, Adab F, Jefferies SJ, Scrase C, Yap BK, A'Hern RP, Sydenham MA, Emson $\mathrm{M}$ and Hall E: Parotid-sparing intensity modulated versus 
conventional radiotherapy in head and neck cancer (PARSPORT): A phase 3 multicentre randomised controlled trial. Lancet Oncol 12: 127-136, 2011. PMID: 21236730. DOI: 10.1016/S1470-2045(10)70290-4

26 Sanguineti G, Endres EJ, Gunn BG and Parker B: Is there a "mucosa-sparing" benefit of IMRT for head-and-neck cancer? Int J Radiat Oncol Biol Phys 66: 931-938, 2006. PMID: 17011465. DOI: $10.1016 /$ j.ijrobp.2006.05.060

27 Sanguineti G, Sormani MP, Marur S, Gunn GB, Rao N, Cianchetti M, Ricchetti F, McNutt T, Wu B and Forastiere A: Effect of radiotherapy and chemotherapy on the risk of mucositis during intensity-modulated radiation therapy for oropharyngeal cancer. Int J Radiat Oncol Biol Phys 83: 235-242, 2012. PMID: 22104358. DOI: $10.1016 /$ j.ijrobp.2011.06.2000

28 Dean JA, Welsh LC, Gulliford SL, Harrington KJ and Nutting CM: A novel method for delineation of oral mucosa for radiotherapy dose-response studies. Radiother Oncol 115: 6366, 2015. PMID: 25779721. DOI: 10.1016/j.radonc.2015.02.020

29 Tasli ML, Mat C, De Simone C and Yazici H: Lactobacillus lozenges in the management of oral ulcers of Behcet's syndrome. Clin Exp Rheumatol 24: 83-86, 2006. PMID: 17067433

30 Sonalika WG, Amsavardani Tayaar S, Bhat KG, Patil BR and Muddapur MV: Oral microbial carriage in oral squamous cell carcinoma patients at the time of diagnosis and during radiotherapy - a comparative study. Oral Oncol 48: 881-890, 2012. PMID: 22513209. DOI: 10.1016/j.oraloncology. 2012.03.018
31 Stokman MA, Spijkervet FK, Burlage FR, Dijkstra PU, Manson WL, de Vries EG and Roodenburg JL: Oral mucositis and selective elimination of oral flora in head and neck cancer patients receiving radiotherapy: A double-blind randomised clinical trial. Br J Cancer 88: 1012-1016, 2003. PMID: 12671696. DOI: $10.1038 /$ sj.bjc.6600824

32 Wijers OB, Levendag PC, Harms ER, Gan-Teng AM, Schmitz PI, Hendriks WD, Wilims EB, van der Est $\mathrm{H}$ and Visch LL: Mucositis reduction by selective elimination of oral flora in irradiated cancers of the head and neck: A placebo-controlled double-blind randomized study. Int J Radiat Oncol Biol Phys 50: 343-352, 2001. PMID: 11380220.

33 Khaw A, Logan R, Keefe D and Bartold M: Radiation-induced oral mucositis and periodontitis - proposal for an interrelationship. Oral Dis 20: e7-e18, 2014. PMID: 24724985. DOI: 10.1111/odi.12199

34 Khaw A, Liberali S, Logan R, Keefe D and Bartold PM: Influence of periodontitis on the experience of oral mucositis in cancer patients undergoing head and neck radiotherapy: a pilot study. Support Care Cancer 22: 2119-2125, 2014. PMID: 24647487. DOI: $10.1007 / \mathrm{s} 00520-014-2186-3$

Received January 21, 2019

Revised February 28, 2019

Accepted March 4, 2019 